\title{
Variabilidad temporal de aerosoles atmosféricos en Huancayo
}

\author{
Temporal variability of atmospheric aerosols in Huancayo
}

Sthefany Virginia Vivanco Cristobal

Universidad Continental

\section{RESUMEN}

Objetivos: Identificar la variabilidad temporal de los aerosoles atmosféricos en Huancayo a través de datos satelitales. Métodos: Investigación de al cance descriptivo, diseño longitudinal. Los datos utilizados fueron, el índice de aerosol (IA) tomados por el sensor $\mathrm{OMl}$ (ozone monitoring instrument) en el período 2005-2012; y el espesor óptico de aerosol (EOA) registrados por el sensor MODIS (moderate resolution imaging spectroradiometer) de las plataformas Aqua y Terra, en los períodos 20032012 y 2001-2012, respectivamente. Resultados: El IA registró el año 2012 un máximo interanual de 0,61 ; durante el período enero-marzo disminuyó a 0,30; abril-agosto aumentó a 0,75 y setiembrediciembre disminuyó a 0,43 . En cambio, el EOA reportó en el 2005 un máximo interanual de 0,22; en el período abril-junio disminuyó a 0,09; juliosetiembre aumentó a 0,30; octubre-diciembre disminuyó a 0,22, y enero-marzo aumentó a 0,20 . El análisis estadístico reportó un coeficiente de correlación entre el IA del sensor OMl y el EOA del sensor MODIS de las plataformas Aqua y Terra, siendo 0,1041 y 0,0982 $(p<0,05)$, respectivamente. La misma correlación fue efectuada entre los datos del sensor MODIS, resultando más elevada 0,902 $(p<0,05)$. El IA mostró una tendencia de incremento a razón de 0,036/año; el EOA de disminución, 0,003/año. Conclusiones: Se identificó un patrón de variación significativo entre las estaciones y los meses de ambos parámetros con elevados valores del IA en invierno y otoño, máximos en agosto; el EOA, en primavera y verano, máximos en septiembre.

Palabras clave: Variabilidad temporal, aerosoles atmosféricos, índice de aerosol, espesor óptico de aerosol. 


\section{ABSTRACT}

Objectives: To identify the temporal variability of atmospheric aerosols in Huancayo through satellite data. Methods: Descriptive scope research, longitudinal design. The used data were, the aerosol index (Al) taken by the OMl (ozone monitoring instrument) sensor in the 20052012 period; and aerosol optical depth (AOD) recorded by the MODIS (moderate resolution imaging spectroradiometer) sensor of Aqua and Terra platforms, in the 2003-2012 and 2001-2012 periods respectively. Results: The $\mathrm{Al}$ recorded a year-on-year maximum of 0,61 on 2012; during the january-march period decreased to 0,30 ; april-august increased to 0,75 and september-december decreased to 0,43. Instead, the EOA reported in 2005 a yearon-year maximum of 0,22 ; in the april-june period decreased to 0,09 ; july-september increased to 0,30 ; October-december decreased to 0,22, and january-march increased to 0,20 . The statistical analysis showed a correlation coefficient between the IA from OMI and EOA from MODIS sensor in Aqua and Terra platforms, being 0,1041 and $0,0982(p<0,05)$, respectively. The same correlation was made between the MODIS sensor data, resulting higher $0,902(p<0,05)$. The IA showed a tendency to increase at the rate of 0,036 /year; EOA decreased 0,003/year. Conclusions: The significant variation pattern was identified between the seasons and the months of both parameters with high IA values in winter and fall, the highest in august; the EOA in spring and summer, highest in september.

Keywords: Temporal variability, atmospheric aerosols, aerosol index, aerosol optical thickness.

\section{INTRODUCCIÓN}

Los aerosoles son partículas sólidas y líquidas que se encuentran suspendidas en la atmósfera, con tamaños que oscilan entre centenas y milésimas de micras. Algunos aerosoles provienen de la combustión industrial, incendios forestales, fuentes de origen natural como la sal del rocío marino, el polvo de los desiertos, o erupciones volcánicas (1).

La caracterización de las variaciones espaciales y temporales de la contaminación por aerosoles es fundamental para un entendimiento completo de su formación, el transporte y la acumulación en la atmósfera (2). Esta información es importante para el control y vigilancia de la variación de los aerosoles y realizar un seguimiento de los acontecimientos que alteran sus concentraciones. De esta manera, su estudio ayudará a una mejor comprensión de los efectos de los aerosoles en el clima, la calidad del aire y la salud.

Del tema de investigación existen pocos estudios específicos; sin embargo, sí se encuentran trabajos relacionados con el tema, que utilizan datos satelitales de aerosoles y que facilita su comprensión del estudio.

La variación espacial y temporal del espesor óptico de los aerosoles sobre Perú, muestra series de tiempo del espesor óptico a diferentes longitudes de onda, y de diferentes sensores desde la plataforma Giovanni. Se observó un comportamiento cíclico, con máximos valores en los meses de verano. Además, se determinó una alta correlación entre los datos adquiridos provenientes de los sensores MODIS, a bordo de los satélites Aqua y Terra (3).

El estudio de la dinámica del espesor óptico de los aerosoles en América del Sur, a partir de imágenes satelitales del sensor MODIS, indica que los datos del espesor óptico de aerosol (EOA) muestra valores máximos en los meses de agosto y septiembre, durante un período de doce años (2001-2012). Además este aumento coincide con el inicio de incendios, generado por los agricultores en la región amazónica, que es muy común durante los meses anteriores (4).

Las emisiones de la quema de biomasa son importantes fuentes de aerosoles y oxidantes químicos, como CO, NOX y partículas de carbono orgánico y elemental (5). La quema 
de biomasa por incendios es una práctica común en América del Sur, inclusive es la mayor fuente de contaminación atmosférica a escala continental (6). En América del Sur, durante la temporada de quema de biomasa la columna de humo cubre un área de unos 4 a 5 millones de $\mathrm{m} 2$ que se ha observado con frecuencia, a través de sensores remotos (7).

Se tiene evidencias del transporte transfronterizo de contaminantes del aire en la Amazonía del Perú debido a las quemas de vegetación en Brasil, donde se evaluó datos de ozono troposférico, así como de aerosoles mediante la teledetección, usando técnicas de los sensores TOMS (total ozone mapping spectrometer) y MODIS. Se pudo establecer la significancia estadística del efecto de las quemas en los niveles de ozono troposférico registrados en Huancayo, los aerosoles mostraron similar tendencia (8).

En la región Delta del río Perla (una de las principales zonas económicas de China) se estudió los efectos de la quema de biomasa en los aerosoles y la concentración del ozono, mediante datos satelitales, mediciones en tierra y análisis de vientos. Los resultados sugieren que las concentraciones elevadas de aerosol debido a las quemas de biomasa producen reducción de la intensidad de UV (radiación ultravioleta) y por lo tanto, decrece la formación de ozono troposférico (9).

En el estudio de la variación estacional de aerosoles transportados a la ciudad de La Paz se encontró fuertes indicios de que las fuentes de aerosoles que llegan y contaminan la región de estudio, provienen de las quemas de biomasa en la Amazonía boliviana y/o brasileña, que son transportados hacia la región del altiplano por los vientos del Este y los sistemas de alta presión en la atmósfera superior. Este transporte es favorecido por la mayor velocidad de los vientos característicos de estos meses y además porque las precipitaciones están reducidas, lo cual genera condiciones atmosféricas favorables para el transporte (10).
La precipitación mensual climatológica en la cuenca del Mantaro para el período 1971 2000 muestra que la distribución anual de lluvias se divide en inicio (septiembrediciembre) y pico (enero-marzo) (11).

Pero, ¿̇cómo es el comportamiento de la variación temporal del espesor óptico de los aerosoles atmosféricos en Huancayo? Este es el problema que da origen a nuestro estudio.

El objetivo general fue identificar la variabilidad temporal de los aerosoles atmosféricos en Huancayo mediante datos satelitales, tomando como parámetros de medición al espesor óptico de aerosol (EOA) y al índice de aerosol (IA), usando datos satelitales procedentes de los sensores MODIS (espectroradiómetro de imágenes de media resolución) y $\mathrm{OMI}$ (instrumento de monitoreo de ozono).

La hipótesis plantea que la variabilidad temporal de los aerosoles atmosféricos en Huancayo muestra un comportamiento estacional.

La investigación permitió obtener información de la presencia de aerosoles en Huancayo, mediante la teledetección que hace posible el monitoreo de la evolución temporal y con un alcance espacial global. Durante la investigación se identificó la necesidad de utilizar datos de sistemas de medición terrestre para la validación de los datos satelitales; asimismo, se identificó que un análisis de trayectorias de precipitación y de campos de vientos ayudaría a una mejor comprensión en relación con la quema de biomasa.

\section{MATERIAL Y MÉTODOS}

El alcance fue descriptivo y diseño longitudinal. Para la recolección se eligió a dos parámetros de estudio más importantes: el espesor óptico de aerosol (EOA) y el índice de aerosol (IA). El estudio toma como referencia a Huancayo, de latitud: -12,05 y longitud: -75,32; en el área está comprendida por las siguientes 
coordenadas geográficas $\mathrm{N}:-10,55, \mathrm{~S}$ : $-13,55, E:-73,32$ y W: $-76,32$.

\section{Análisis del EOA}

Para el análisis del espesor óptico de aerosol (EOA) fueron recolectados datos del sensor MODIS, desde las plataformas Aqua y Terra, en los períodos 2003-2012 y 2001 -2012 , respectivamente.

El sensor MODIS (espectroradiómetro de imágenes de media resolución), vuela a bordo de los satélites Aqua (figura 1) y Terra (figura 2) del Sistema de Observación de la Tierra (EOS) de la NASA. Fue estudios avanzados de la Tierra, el océano, y propiedades de la atmósfera, tales como cobertura de nubes, perfiles atmosféricos, propiedades de los aerosoles, precipitación total de agua y propiedades de nubes (12).

Los productos operacionales de MODIS son generados en tres niveles: nivel $1-L 1$, nivel 2-L2 y nivel 3-L3. El producto de aerosoles MOD04 y MYDO4 es generado a partir del nivel 2-L2. Este sensor proporciona información diaria del contenido de aerosoles: el exponente de Ångström $(\alpha)$, espesor óptico de aerosoles $\left(T_{A}\right)$, albedo de dispersión simple $\left(\omega_{0}\right)$, radio efectivo $(r)$, parámetro de asimetría (g) y otros (13).

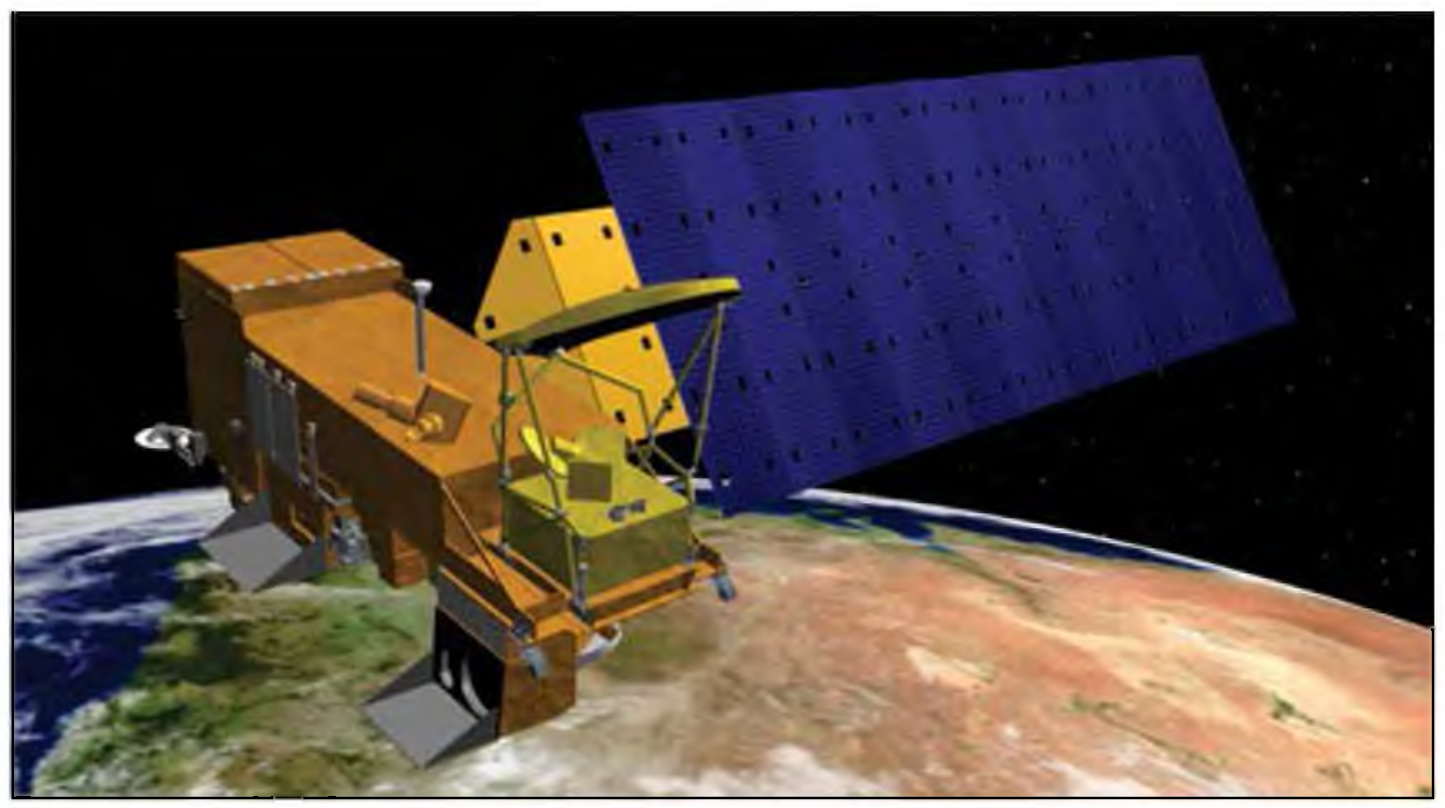

Figura $\mathrm{N}^{\circ}$ 1: Plataforma Aqua.

Fuente $\quad:$ hHp://spaceplace.nasa.gov/review/earth-card-game/\#

lanzado por esta administración a bordo de Terra en 1999 y a bordo de Aqua en el 2002. Explora un ancho de franja de 2 $330 \mathrm{~km}$, que es lo suficientemente amplia como para proporcionar cobertura global casi completa cada dos días desde la órbita polar, sincronizada con el Sol, con plataforma a una altitud de 705 $\mathrm{km}$. MODIS proporciona imágenes en 36 bandas espectrales entre 0,415 y 14 $235 \mu \mathrm{m}$; con resoluciones espaciales de $250 \mathrm{~m}$ (2 bandas), $500 \mathrm{~m}$ (5 bandas) y $1000 \mathrm{~m}$ (29 bandas). Estas bandas han sido cuidadosamente seleccionadas para
Del conjunto de parámetros que proporciona MODIS, este estudio utilizó datos del espesor óptico de aerosol (EOA) sobre la tierra a $550 \mathrm{~nm}$. Estos valores se promediaron tomando los pixeles próximos a las coordenadas geográficas de Huancayo en una rejilla de $\pm 1,5^{\circ}$.

El espesor óptico de aerosol (TA) es una magnitud adimensional que indica el poder que poseen las partículas de aerosol para atenuar la radiación una cierta longitud de onda (14). Otros autores señalan que el EOA es el grado en que los aerosoles 
advierten la transmisión de la luz por absorción o dispersión de la luz (15). Es importante mencionar que cuanto mayor sea el espesor óptico a una determinada longitud de onda, menos luz de esa longitud de onda alcanzará la superficie de la Tierra. La medición del espesor óptico de los aerosoles en más de una longitud de onda puede proporcionar información importante sobre la concentración, distribución del tamaño y la variabilidad de los aerosoles en la atmósfera.

\section{Análisis del IA}

Para el análisis del índice de aerosol (IA)
$\mathrm{NO} 2, \mathrm{HCHO}, \mathrm{BrO}$ y $\mathrm{OClO}$, además mide características del aerosol, cobertura de nubes y radiación UV en la superficie (16).

Los productos operacionales del OMI son: albedo de dispersión simple, extinción cerca del UV, profundidad óptica de absorción (17).

De los parámetros que proporciona $\mathrm{OMI}$, este estudio utilizó datos sobre el índice de aerosol UV (IA) a $360 \mathrm{~nm}$. Estos valores se promediaron tomando los pixeles próximos a las coordenadas geográficas de Huancayo en una rejilla de $\pm 1,5^{\circ}$.

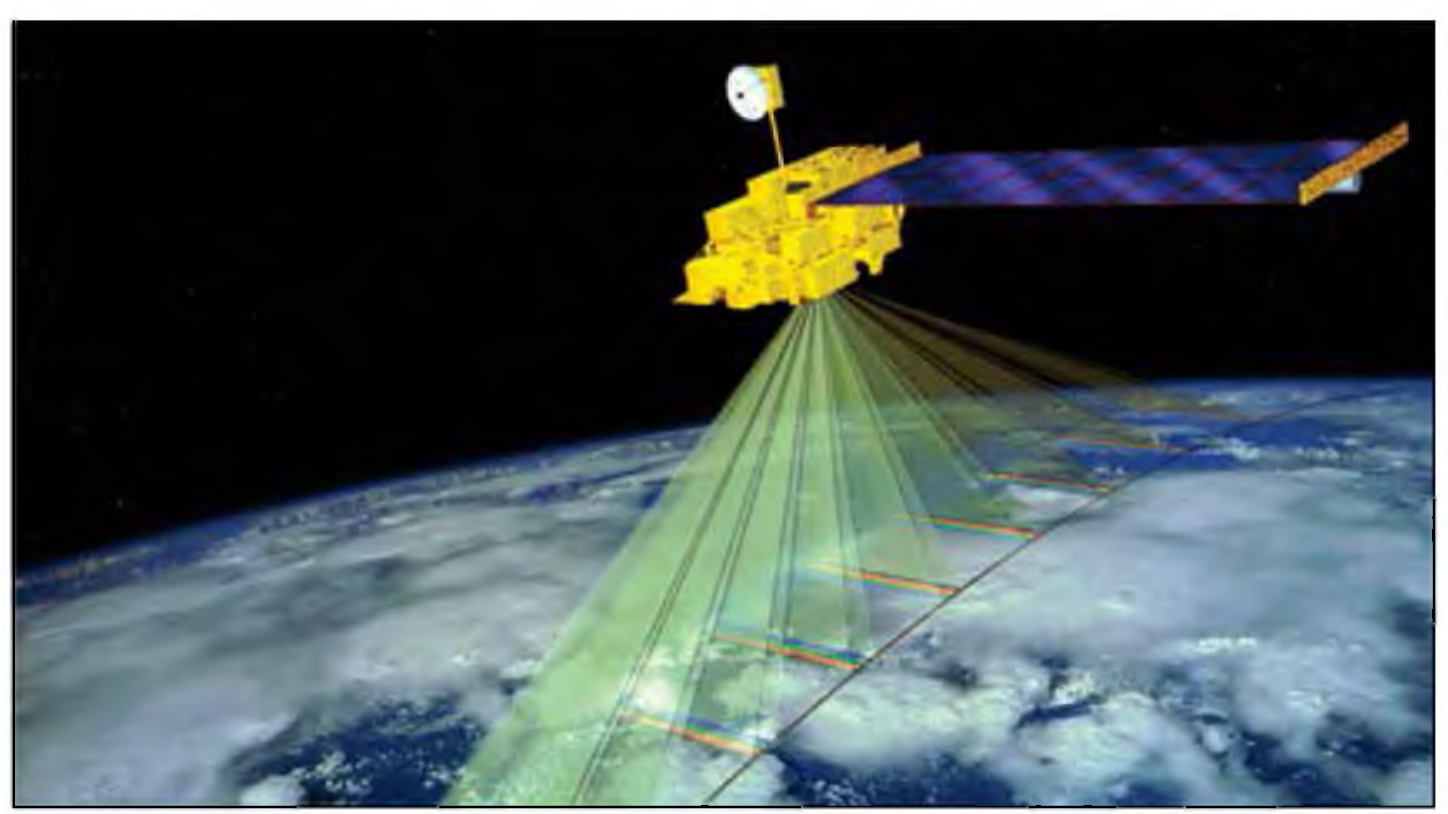

Figura $\mathrm{N}^{\circ}$ 2: Plataforma Terra.

Fuente $\quad:$ hHp://spaceplace.nasa.gov/review/earth-card-game/\#

fueron recolectados datos del sensor $O M I$ en el período 2005-2012.

OMI (instrumento de monitoreo de ozono) es un sensor lanzado en el 2004 a bordo del satélite Aura (figura 3) del Sistema de Observación de la Tierra de la NASA; explora una franja de $2600 \mathrm{~km}$ que permite realizar mediciones con una cobertura global diaria. Con resolución espacial de $13 \mathrm{~km} \times 24 \mathrm{~km}$ puede ampliarse hasta 13 $\mathrm{km} \times 12 \mathrm{~km}$, lo que permite la detección de la contaminación del aire a escala urbana. Los gases traza medidos son $\mathrm{O} 3$,
El índice de aerosol UV (IA) es la diferencia entre las observaciones y los cálculos de los modelos de absorción y coeficientes de absorción de radiación no espectrales.

Los valores positivos del índice de aerosol representan generalmente aerosoles absorbentes (polvo y humo), mientras que los valores negativos representan nubes $y$ aerosoles no absorbentes.

El índice de aerosol puede ser interpretado en términos de la profundidad óptica del índice de refracción, distribución del 


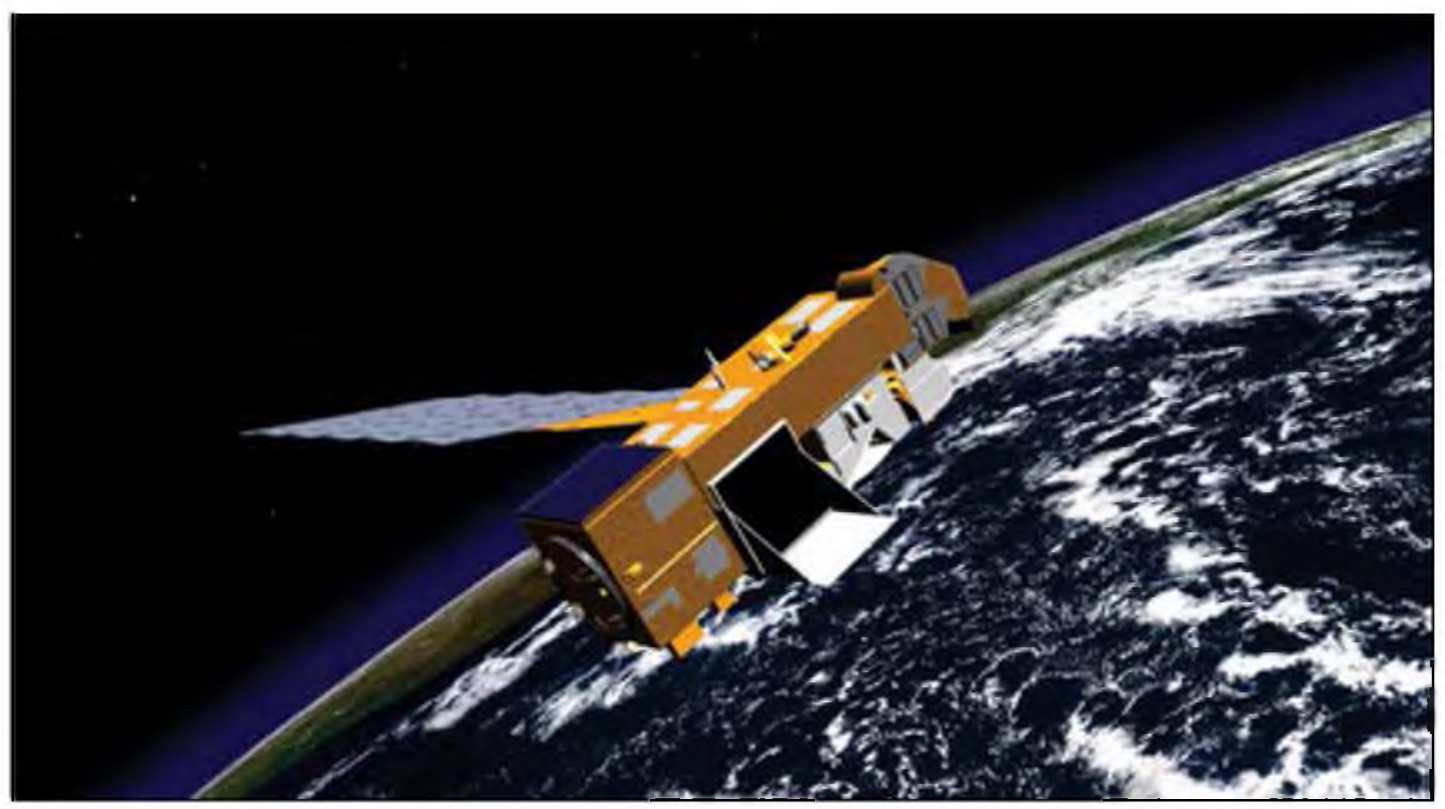

Figura $N^{\circ}$ 3: Plataforma Aura.

Fuente $\quad:$ http://spaceplace.nasa.gov/review/earth-card-game/\#

tamaño de partícula, y la altura de la capa de aerosol (15). Para el tratamiento de datos se utilizó los valores positivos del IA.

El análisis estadístico comprendió el tratamiento descriptivo (medidas de tendencia central y dispersión) e inferencial (análisis de correlación y regresión, prueba F, prueba de Kruskal-Wallis).

\section{RESULTADOS}

\section{Variación interanual}

La figura 4 muestra los datos diarios del IA (adimensional) registrados por $\mathrm{OMI}$ durante 8 años (desde el 1 de enero de 2005 hasta el 31 de diciembre de 2012). Se puede apreciar que el patrón de comportamiento es el mismo, es decir registra máximos

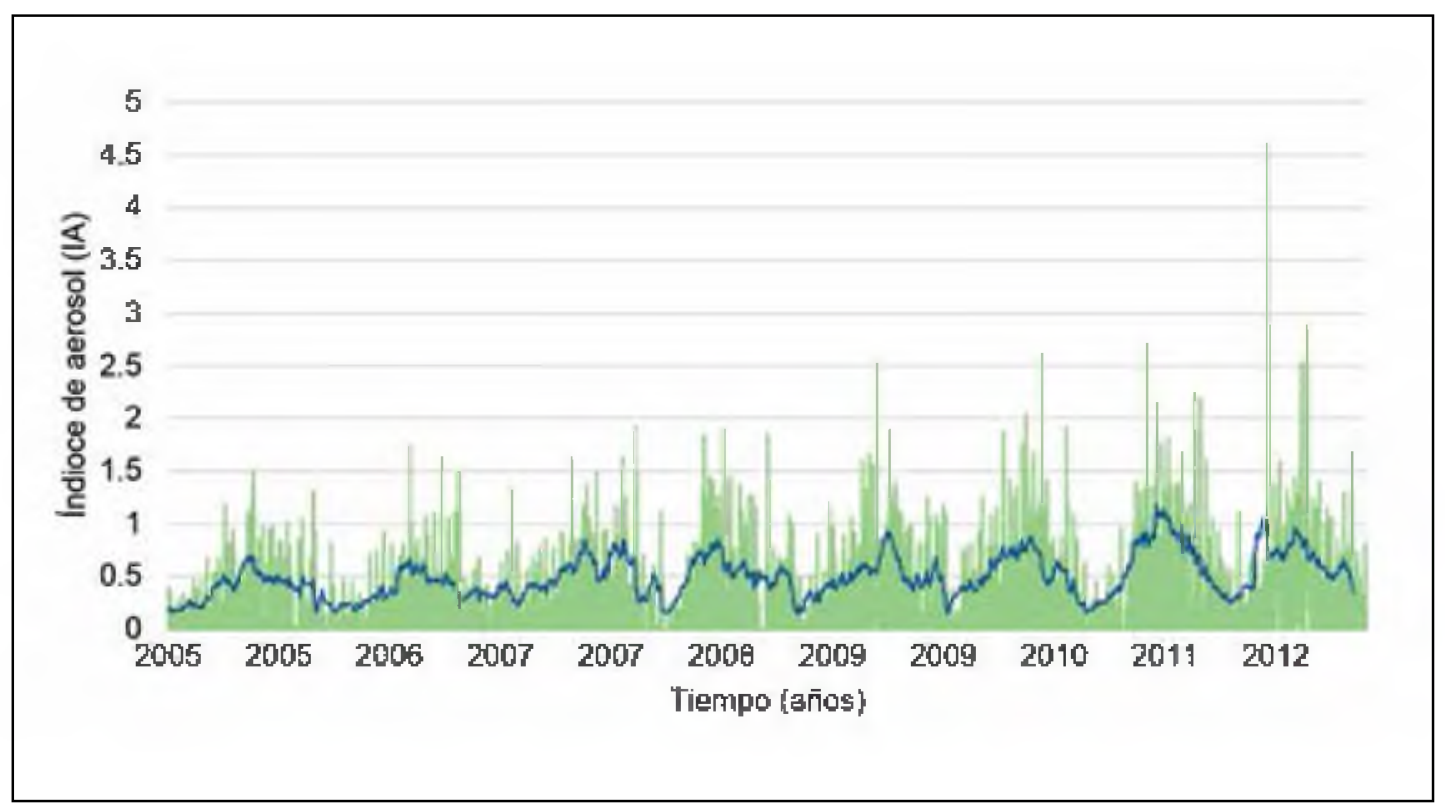

Figura $\mathrm{N}^{\circ}$ 4: Datos diarios del IA registrados por $\mathrm{OMI}$. 


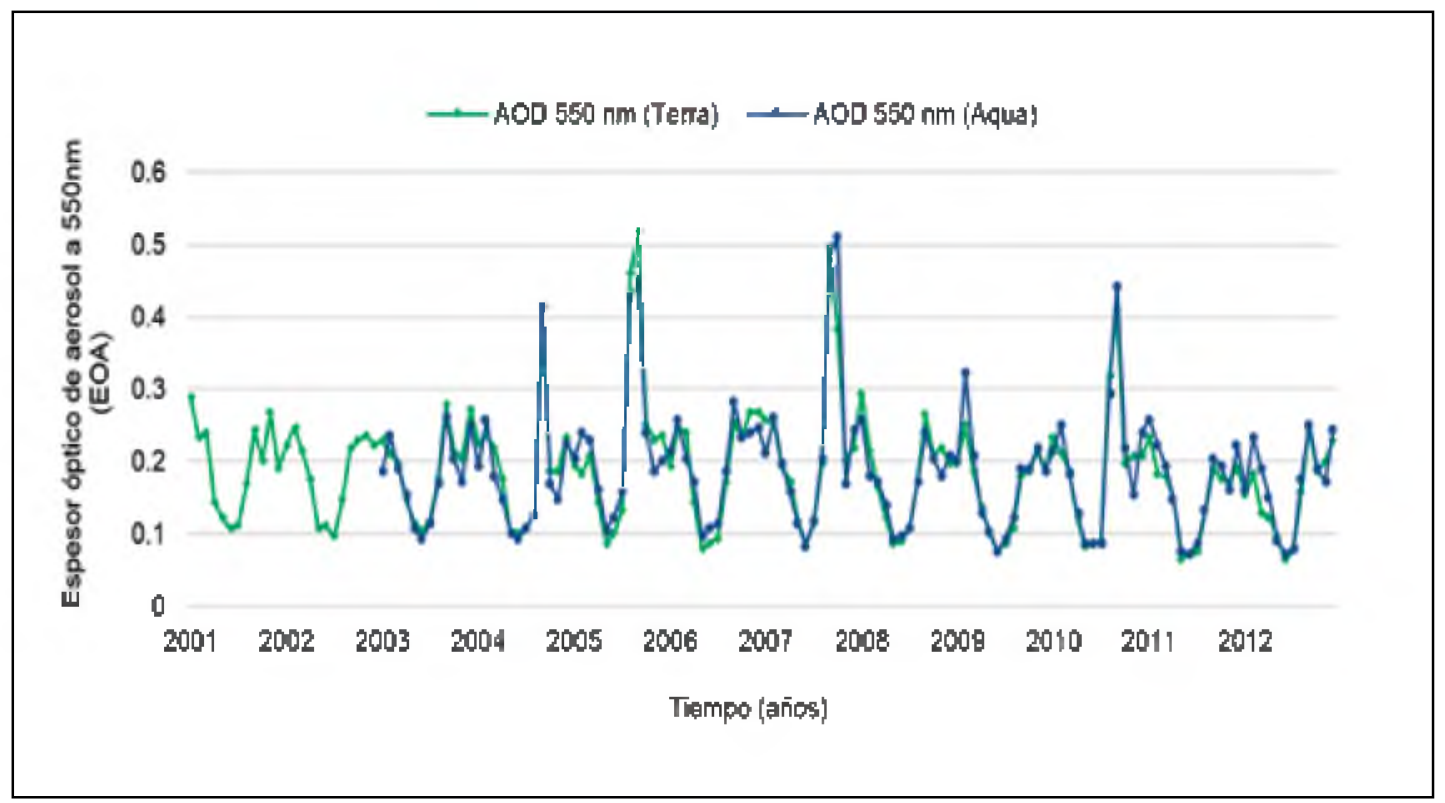

Figura $N^{\circ}$ 5: Variabilidad interanual del EOA550 con el sensor MODIS de las plataformas Aqua y Terra, a lo largo de 10 años y 12 años, respectivamente.

y mínimos en las mismas épocas y con la misma periodicidad. Este hecho está claramente reflejado por la media móvil de cada punto (representado de color azul en la figura 4). Se obtuvo un promedio de $0,51 \pm 0,44$ (prom. \pm desvep.) Los límites de variabilidad del IA no superan el valor de 5 . El valor máximo es de 4,61 que se dio el 30 de abril del 2012.

La figura 5, muestra la evolución interanual del EOA550 (adimensional) medida por el sensor MODIS de las plataformas Aqua y Terra durante 10 y 12 años, respectivamente. Ambas series de tiempo están definidas por ascensos y descensos, de manera repetitiva durante todos los años. Con promedio de $0,18 \pm 0,14$ los valores del EOA tienen máximos de 0,51 en septiembre de 2005 y octubre de 2007, y mínimos de 0,06 y 0,07 en junio de 2012.



Figura $\mathrm{N}^{\circ}$ 6: Diagrama de cajas y bigotes mensuales del índice de aerosol. 




Figura $N^{\circ}$ 7: Diagrama de cajas y bigotes mensuales del espesor óptico de aerosol a $550 \mathrm{~nm}$ del sensor MODIS, desde las plataformas Aqua y Terra.

\section{Variación mensual}

A partir de la observación del comportamiento periódico durante todos los años de las figuras 4 y 5 , se ha representado las figuras 6 y 7 , con diagramas de caja de manera mensual, que incluye la media y la mediana del IA y $\mathrm{EOA}_{550}$.

La figura 6, muestra la evolución del IA. Se observa un incremento periódico en invierno y otoño, y una disminución en primavera y verano. Durante enero, febrero y marzo se registran mínimos con valores inferiores a 0,3 , desde abril hasta agosto se observa un incremento. En el mes de agosto se registra el máximo valor alcanzado durante todo el año con 0,75 $\pm 0,49$. Posterior a estos meses, en septiembre y octubre disminuye hasta 0,5 . Luego experimenta un ligero incremento hasta 0,52 en noviembre $y$ finalmente en diciembre disminuye hasta 0,43 .

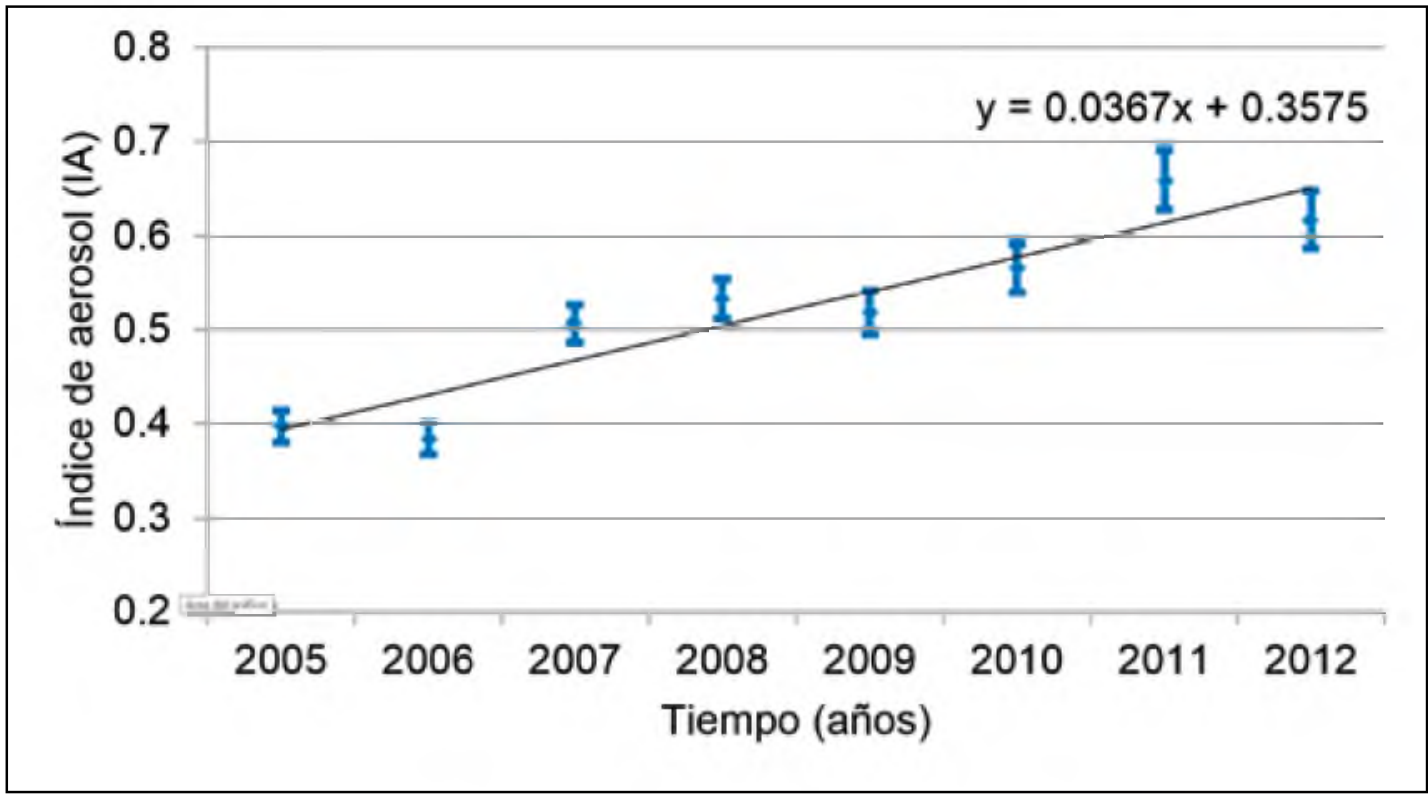

Figura $\mathrm{N}^{\circ}$ 8: Promedios anuales del $\mathrm{IA}_{360}$ con el sensor $\mathrm{OMI}$. 


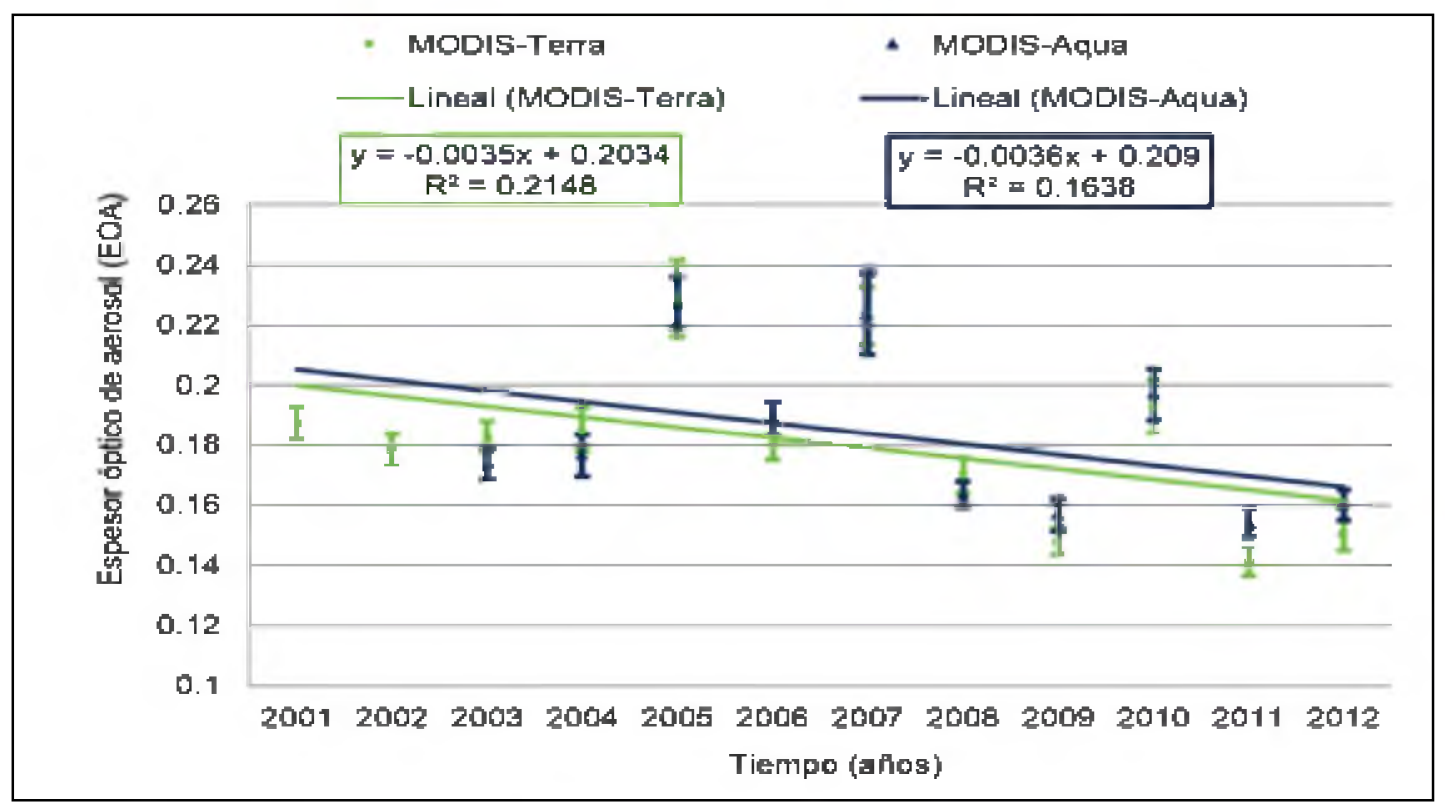

Figura $\mathrm{N}^{\circ}$ 9: Promedios anuales del $\mathrm{EOA}_{550}$ con el sensor MODIS de las plataformas Aqua y Terra.

La figura 7, representa la variabilidad mensual del EOA550, donde se observa que en primavera y verano se experimenta un incremento, mientras que en otoño e invierno una disminución. Durante abril, mayo y junio disminuye hasta 0,09 , con mínimo anual en junio alcanzando un valor de 0,08 $\pm 0,04$. Posterior a estos meses, desde julio a septiembre, se observa un incremento de hasta 0,30 , en septiembre se registra el máximo anual alcanzando un valor de 0,32 $\pm 0,22$ y 0,30 $\pm 0,24$, para las plataformas Aqua y Terra, respectivamente En octubre, noviembre y diciembre disminuye hasta 0,22 . Finalmente, para enero, febrero y marzo aumenta de manera ligera hasta 0,20.

\section{Análisis de tendencias}

La figura 8 muestra la tendencia del IA, durante un período de ocho años. Se observó un incremento a razón de 0,036/ año, en un 4,75\%/año. Además, en el 2012 registró un valor máximo de 0,6172, y el 2006 un mínimo de 0,3844. En cambio, en la figura 9, las tendencias en el transcurso del período estudiado muestran que el EOA indica una disminución de 0,003/año, en un $0,214 \%$ /año y $3,31 \% / a n ̃ o$, para las plataformas Aqua y Terra, respectivamente.

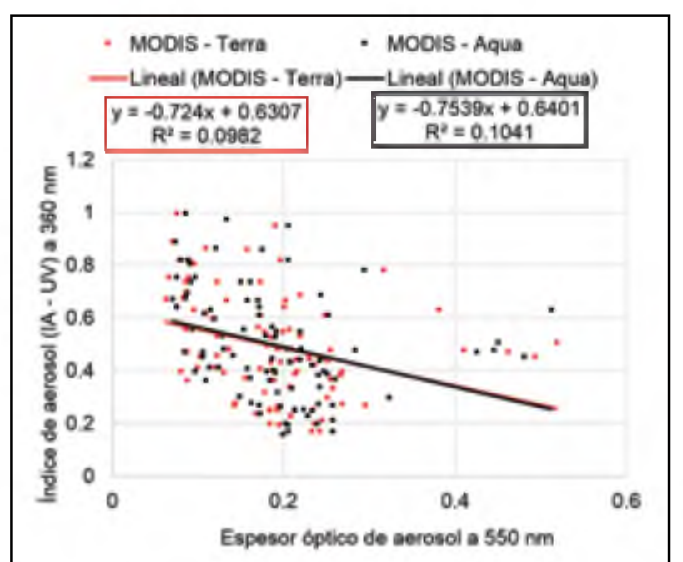

Figura $\mathrm{N}^{\circ}$ 10: Correlación y regresión del índice de aerosol-UV del sensor OMl con el espesor óptico de aerosol de sensor MODIS de las plataformas Aqua y Terra.

También se registró en el 2005 un valor máximo interanual de 0,22, y en el 2011 un mínimo de 0,15.

\section{Análisis de correlación y regresión}

En la figura 10 se observa la correlación entre el IA y el EOA del sensor MODIS de la plataforma Aqua, corresponde al valor de $R 2=0,1041$. Similar resultado se obtuvo en la correlación con el sensor MODIS de la 


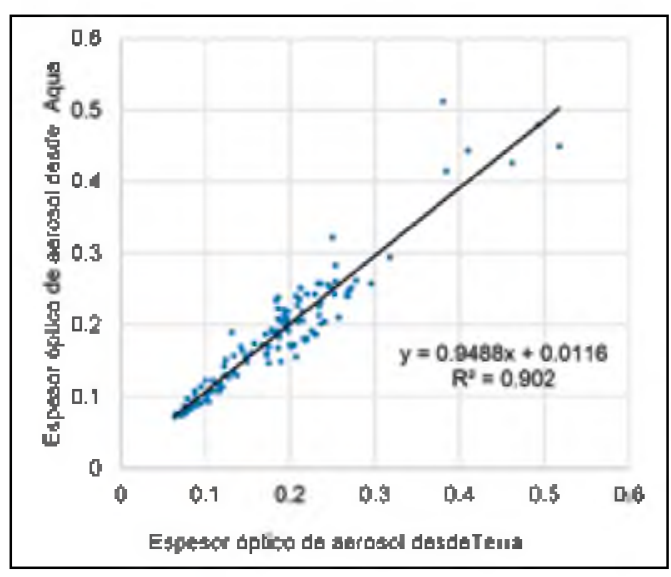

Figura $\mathrm{N}^{\circ}$ 11: Correlación del EOA a $550 \mathrm{~nm}$ medidos por el sensor MODIS de las plataformas Aqua y Terra.

Plataforma Terra, R2 =0,0982.

No obstante, la figura 11 muestra la correlación de los datos de espesor óptico de aerosol, correspondientes al sensor MODIS de las plataformas Aqua y Terra, es alta $(R 2=0,902)$.

\section{DISCUSIÓN}

Con respecto a la variabilidad interanual del EOA550 en Huancayo (figura 5), se ha determinado que el máximo corresponde a septiembre del 2005 con valor de 0,51 en comparación con Rojas et al. (3) da como resultado 0,32 en septiembre del 2005, en un estudio realizado en Perú, con datos de los satélites Aqua y Terra, esta diferencia de valores es debido a que el área geográfica de estudio tuvo mayor cobertura espacial. En otro estudio (4), se reporta que las series de tiempo del EOA550 muestran valores máximos en los meses de agosto y septiembre, en América del Sur, durante el período 2000-2012.

La variabilidad mensual del IA (figura 6), registra el máximo valor de 0,7504 \pm 0,4942 en agosto, mientras que Suárez et al. (8) reportan también el mismo mes, con un valor aproximado a 0,72 .

Para la variabilidad mensual (figura 7), se observa que en enero, febrero y marzo el EOA550 aumenta; para abril, mayo y junio, disminuye; para julio, agosto y septiembre, vuelve a aumentar; y por último, para octubre, noviembre y diciembre, otra vez disminuye; similar a lo reportado por Rojas et al. (3).

Los resultados de variabilidad mensual del IA y del EOA550 registran como máximo anual a los meses de agosto y septiembre, respectivamente, que podrían estar marcados por el incremento de contaminantes en la Amazonía (8), causado, entre otros factores, por la quema de biomasa (6).

Asimismo se observó que el EOA550, a diferencia del $\mid A$, tiene un segundo máximo anual correspondiente a febrero, casi del mismo orden que en septiembre; sin embargo, no debe confundirse con elevados niveles de aerosol, debido principalmente a dos razones: durante estos meses se forman gran cantidad de nubes que incrementan los valores de los registros del espesor óptico del aerosol; y la otra razón es que en esta época se produce gran cantidad de precipitación (11), que limpia la atmósfera de aerosoles y por lo tanto evita la existencia de estos en grandes cantidades (10). Por esta razón, la baja frecuencia de cobertura de nubes es favorable al monitoreo de aerosoles por sensores en satélites (18).

Las tendencias muestran que el IA tiende al incremento a razón de 0,036/año (figura 8); mientras que el EOA, a la disminución, de 0,003/año (figura 9). Los datos recuperados de los satélites pueden capturar la naturaleza sinóptica de eventos de contaminación y ayudar a identificar las fuentes de los contaminantes a gran escala; sin embargo, se necesita más estudios sobre la forma de utilizar mejor los datos a fin de tener una mejor comprensión de los mecanismos de formación y transporte (2).

El análisis de la correlación entre el IA y el EOA550 (figura 10) es menor a 0,105 debido a que el $\mathrm{IA}$ se midió en la región ultravioleta $(360 \mathrm{~nm})$, y el EOA550 en la región visible $(550 \mathrm{~nm})$. Esta baja correlación pudo darse debido a que los datos se midieron en distintas regiones de 
la radiación solar para su evaluación.

En cambio, el análisis de los datos de EOA550, procedentes del sensor MODIS, de los satélites Aqua y Terra, reportó un coeficiente de correlación alto, con $\mathrm{R} 2=0,902$ (figura 11), lo que indica una buena relación. En el estudio de Rojas et al (3), la correlación alcanzó a R2=0,935.

Durante el año el IA muestra una época comprendida por valores máximos en los meses de abril-septiembre (figura 7). Esta característica podría estar asociada al período seco (estiaje), en el que las precipitaciones se ven reducidas (11).

Para el análisis estadístico de todos los datos, se ejecutó la prueba $F$, puesto que el valor $P$ es menor que 0,05 , existe una diferencia estadísticamente significativa entre las medias de las variables con un nivel del 95,0\% de confianza. Luego se aplicó la prueba de Kruskal-Wallis, resultó el valor $P$ menor que 0,05 , existe una diferencia significativa entre las medianas con un nivel del $95,0 \%$ de confianza. No obstante, estos datos deben ser validados y recuperados con datos en tierra, desarrollando metodologías claras y precisas que mejoren su comprensión.

El trabajo permitió estudiar la variabilidad del EOA y IA en Huancayo, con la finalidad de dar paso a nuevas investigaciones que realicen un seguimiento de los acontecimientos que alteran sus concentraciones e identificar las fuentes de origen. Esta información puede ser utilizada en la planificación de la gestión de la calidad aire que incluya la implementación de una red de monitoreo de la contaminación atmosférica y medidas de control.

Los datos obtenidos evidencian un comportamiento estacional, es decir un patrón de variación significativa de la concentración de aerosoles que presenta elevados valores del IA en invierno $y$ otoño, con máximos en agosto; el EOA, en primavera y verano, y máximos en septiembre. Este comportamiento es repetitivo durante todo el período estudiado.

\section{Agradecimientos}

Por el financiamiento de este proyecto a la National Academy of Sciences de los Estados Unidos (NAS) y la United State Agency International for Development (USAID). Asimismo a la dirección de investigación de la Universidad Continental por la asesoría en el proceso de investigación.

\section{REFERENCIAS BIBLIOGRÁFICAS}

1. Seinfeld J, Pandis S. Atmospheric chemistry and physics from Air pollution to climate change. $1^{\mathrm{a}}$ ed. New York: Wiley; 1998.

2. Zheng J, Che W, Zheng Z, Chen L, Zhong L. Analysis of spatial and temporal variability of $\mathrm{PM} 10$ concentrations using MODIS aerosol optical thickness in the Pearl River Delta Region, China. Aerosol and Air Quality Research. 2013; 13: 862-876.

3. Rojas J, Vargas P, Carrillo F. Variabilidad espacial y temporal del Espesor Óptico de los Aerosoles sobre Perú usando Imágenes de Satélite. Lima: Laboratorio de Teledetección/UNMSM; 2009.

4. Rojas N, Rojas J. Estudio de la Dinámica del Espesor Óptico de los Aerosoles en América del Sur a partir de las imágenes MODIS de los satélites Terra y Aqua (2000-2012). Lima: Laboratorio de Teledetección/UNMSM; 2012.

5. Galanter $M$, Levy $H$, Carmichael $G$. Impacts of biomass burning on tropospheric $\mathrm{CO}, \mathrm{NOx}$ and $\mathrm{O}$, J. Geophys. Res. 2000; 105 (D5): $6633-$ 6653.

6. Mielnicki D, Canziani P, Drummond J, Skalany JP Quema de biomasa en el Centro-Sur de Sudamérica: Incendios locales, impactos regionales. [Internet]. 2005 Noviembre [citado 2014 Enero 10]: [10 páginas]. Disponible en: http://www.uca.edu.ar/uca/common/ grupo72/files/QUema_biomasa_ centro_sur_sudamerica.pdf

7. Prins $\bar{E}$, Feltz J, Menzel W, Ward D. An overview of GOES-8 diurnal fire and 
smoke results for SCARB and 1995 fire season in South America. J. Geophys. Res. 1998; 103(D24), 31821-31835.

8. Suárez L, Castillo L, Marín M, Carrillo G, Rímac L, Pomalaya J, et al. Estudio de la variación estacional del ozono troposférico y aerosoles del Perú relacionado a las quemas de vegetación en la Amazonía. Mosaico Cient. 2006; 3(2): 36-41.

9. Deng X, Tie X, Zhou X, Wu D, Zhong L, Tan $H$, et al. Effects of Southeast Asia biomass burning on aerosols and ozone concentrations over the Pearl River Delta (PRD) region. Atmospheric Environment. 2008; 42: 8493-8501.

10. Velarde F. Estudio de la Variación Estacional de Aerosoles Transportados a la ciudad de La Paz y su relación con las quemas de Biomasa. [Tesis de grado]. La Paz: Universidad Mayor de San Andrés, Facultad de Ciencias Puras y Naturales; 2010.

11. Silva Y, Takahashi K, Chávez R. Dry and wet rainy seasons in the Mantaro river basin (Central Peruvian Andes). Adv. Geosci. 2007; 14: 1-4.

12. King MD, Menzel WP, Kaufman YJ, Tanré D, Gao BC, Platnick S, et al. Cloud and aerosol properties, precipitable water, and profiles of temperature and water vapor from MODIS. IEEE Transactions on Geoscience and Remote Sensing. 2003; 41 (2): 442-458.

13. Levy RC, Lorraine A, Remer, Mattoo S, Vermote EF, Kaufman YJ. Secondgeneration operational algorithm: Retrieval of aerosol properties over land from inversion of Moderate Resolution Imaging Spectroradiometer spectral reflectance. J. Geophys. Res. 2007; 112(D13211): 1-21.

14. Expósito F. Determinación del espesor óptico de aerosoles mediante técnicas de teledetección en la región de Canarias. [Tesis Doctoral]. Mallorca: Universidad de la Laguna; 1999.

15. GES DISC Web [Internet]. New York: National Aeronautics and Space Administration; [update 2009 Sep 08; cited 2013 Oct 31]. About MODIS [about 3 screens]. Available from: http:// disc.sci.gsfc.nasa.gov/dataholdings/PIP
16. Levelt $P$, Hilsenrath $E$, Leppelmeier $G$, Van Den Oord G, Bhartia P, Tamminen $J$, et al. Science objectives of the Ozone Monitoring Instrument. IEEE Transactions on Geoscience and Remote Sensing. 2006; 44(5): $1199-1208$.

17. Reyes P Análisis y descripción general de la Plataforma GIOVANNI: Universidad de Granada; 2010.

18. Almeida A. Propriedades ópticas das partículas de aerossol e uma nova metodologia para a obtenção de espessura óptica via satélite sobre São Paulo. [Tese de doutorado]. São Paulo: Instituto de Física para obtenção do título de Doutor em Ciências. Brasil; 2005. 